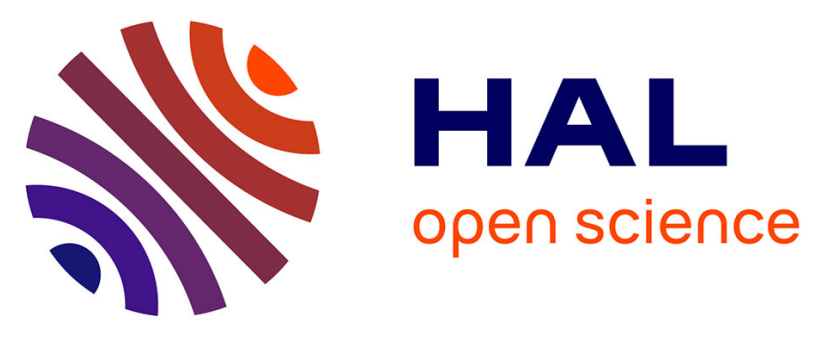

\title{
Polymorphism of CAG motif of SK3 gene is associated with acute oxaliplatin neurotoxicity
}

Michele Basso, Anna Modoni, Daniele Spada, Alessandra Cassano, Giovanni

Schinzari, Mauro Lo Monaco, Davide Quaranta, Pietro Attilio Tonali, Carlo

Barone

\section{To cite this version:}

Michele Basso, Anna Modoni, Daniele Spada, Alessandra Cassano, Giovanni Schinzari, et al.. Polymorphism of CAG motif of SK3 gene is associated with acute oxaliplatin neurotoxicity. Cancer Chemotherapy and Pharmacology, 2010, 67 (5), pp.1179-1187. 10.1007/s00280-010-1466-y . hal00626286

\section{HAL Id: hal-00626286 \\ https://hal.science/hal-00626286}

Submitted on 25 Sep 2011

HAL is a multi-disciplinary open access archive for the deposit and dissemination of scientific research documents, whether they are published or not. The documents may come from teaching and research institutions in France or abroad, or from public or private research centers.
L'archive ouverte pluridisciplinaire HAL, est destinée au dépôt et à la diffusion de documents scientifiques de niveau recherche, publiés ou non, émanant des établissements d'enseignement et de recherche français ou étrangers, des laboratoires publics ou privés. 


\section{Polymorphism of CAG motif of SK3 gene is associated with acute oxaliplatin neurotoxicity}

Basso Michele ${ }^{1}$, Modoni Anna ${ }^{2}$, Spada Daniele ${ }^{1}$, Cassano Alessandra $^{1}$, Schinzari Giovanni ${ }^{1}$, Lo Monaco Mauro $^{2}$, Quaranta Davide ${ }^{2}$, Tonali Pietro Attilio ${ }^{2}$ and Barone Carlo ${ }^{1}$.

${ }^{1}$ Medical Oncology, Catholic University of Sacred Heart, Rome, Italy

${ }^{2}$ Department of Neuroscience, Catholic University of Sacred Heart, Rome, Italy

Running title: SK3 and oxaliplatin neurotoxicity.

Key words: neurotoxicity, oxaliplatin, KCa channels, SK3 gene polymorphism, peripheral nerve excitability.

Disclosures: Carlo Barone was a consultant for Amgen, Merck and Roche

\section{Correspondence to:}

Prof Carlo Barone

Medical Oncology

Department of Internal Medicine

Catholic University of Sacred Heart

Largo Agostino Gemelli n 8

00168 Rome, Italy

Tel: +390630154844

Fax: +39063017334

e-mail: carlobarone@ rm.unicatt.it 


\section{Abstract}

Purpose. There is no agreement on which channel is involved in oxaliplatin neurotoxicity, most investigators favouring voltage-gated sodium channels. However, the small conductance $\mathrm{Ca}^{++}$activated $\mathrm{K}^{+}$channels, encoded by the SK 1-3 genes, are also involved in membrane excitability, playing a role in after-hyperpolarisation at the motor nerve terminal. As the SK3 gene is characterised in Caucasians by a highly polymorphic CAG motif within the exon 1, we hypothesise that SK3 gene polymorphism may influence the development of acute nerve hyperexcitability in oxaliplatin-treated patients.

Methods. Patients eligible for an oxaliplatin-containing regimen were enrolled. Detailed neurological examination, nerve conduction studies and needle electromyography were performed before and after oxaliplatin administration. DNA was extracted by polymerase chain reaction and each allele was isolated and sequenced.

Results. We evaluated 40 patients. After oxaliplatin administration, 28 patients developed symptoms of neurotoxicity, which were severe in eleven. Patients were divided into 3 groups according to neurophysiologic data : G0 (normal peripheral nerve excitability [PNE]), 16 patients; G1 (mild PNE), 15 patients; G2 (severe PNE), 9 patients. Genetic analysis showed different alleles ranging from 13 to 23 CAG repeats. Patients carrying alleles containing 13-15 CAG repeats experienced a significantly higher incidence of severe nerve hyperexcitability (chi-square 48.6; df 16; $p=.0001$ ).

Conclusion. The results suggest that OXA-neurotoxicity may be related to distribution of the polymorphic CAG motif of the SK3 gene, which might modulate nerve after-hyperpolarisation. The 13-14 CAG repeat allele could mark patients susceptible to acute OXA neurotoxicity. 


\section{Introduction}

Oxaliplatin (OXA) is a third-generation derivative of platinum compounds [1]. OXAcontaining regimens are now considered standard therapeutic options for patients with metastatic colorectal cancer [2, 3]. Overall, OXA has a good profile of toxicity but neurotoxicity is the doselimiting side effect. It is closely related to the 1,2 diaminocycloexane carrier ligand which enhances the antitumour activity, but also modifies the neurological toxicity that OXA shares with other platinum compounds $[4,5]$. Cisplatin-related neurotoxicity consists of a chronic dose-dependent distal sensory neuropathy of unknown origin, although damage to gangliar neurons in the dorsal root has been suggested [6]. In addition, patients receiving OXA may develop acute neurological symptoms, such as cold-induced paraesthesia and pharyngo-laryngeal dysaesthesia [7].

Clinical and electrophysiologic studies have shown that OXA gives rise to hyperexcitability at peripheral motor nerves. In particular, in patients developing acute neurotoxicity, a single electrical stimulus to the motor nerves produces a compound muscle action potential (CMAP) followed by repetitive discharges. At the same time, electromyography (EMG) studies show spontaneous high frequency discharges of motor unit multiplets [7, 8]. Overall, these findings are consistent with those observed in neuromyotomia $[9,10]$, an autoimmune disease caused by auto-antibodies against voltagegated potassium channels (VGKC). Despite this well-known observation, the experimental evidence is still conflicting. Indeed, electrophysiologic OXA-induced changes are not mimicked by the VGKC blockers, 4-aminopyridine and tetraethylammonium, suggesting that OXA might not interfere with VGKCs [11]. Other authors have shown that OXA-induced hyperexcitability may be prevented by tetradotoxin (TTX) or carbamazepine, which in turn block voltage-gated sodium channels (VGNaCs). Moreover, $\beta$-pompilidotoxin, which slows $\mathrm{Na}^{+}$channel inactivation, induces changes resembling those observed in OXA neurotoxicity, whereas VGKC blockers such as apamin were not able to reproduce OXA-induced hyperexcitability [12]. Patch-clamp technique studies performed on neurons isolated from the central nervous system of the cockroach showed that OXA was able to alter VGNaCs through 
a pathway involving calcium ions [13]. Taken together, these data are consistent with a channelopathy involving $\mathrm{VGNaCs}[11,12,13]$.

Following the observation that a life-threatening coma during an OXA infusion was related to severe hypokalaemia and hypocalcaemia, we focused our attention on the role of potassium channels in OXAneurotoxicity [14]. SK3 (KCNN3) is a unique type of small-conductance calcium-dependent potassium channel that is highly represented in the peripheral nervous system, but absent from the central nervous system and muscular tissue [15]. Interestingly, a highly polymorphic CAG repeat region, essential for the formation of tetramers, characterizes the gene encoding these channels. Among Caucasians the length of the most common allele containing the CAG motif is about 18-19 repeats, with a range of 1226 [16-18]. Bearing in mind that SK3 channels have a single transmembrane domain, we assumed that interactions between each subunit would be crucial for the efficiency of the channel. Therefore we analyzed a group of patients treated with OXA in order to assess the influence of the genotype, i.e. the polymorphic CAG motif of the SK3 gene, on the development of acute OXA neurotoxicity. 


\section{Patients and methods}

\section{Patients}

Patients aged between 18 and 75 years, eligible for oxaliplatin-containing chemotherapy were enrolled in this study, provided that they had an ECOG performance status lower than 2 and a normal neurophysiologic assessment before OXA administration. A histologically proven diagnosis of colorectal, gastric, pancreatic or biliary cancer was required. All patients had to be OXA naïve, but previous chemotherapy was allowed except for regimens containing cisplatin, taxanes or vinca alkaloids. Patients with symptomatic sensory neuropathy, brain metastases, leptomeningeal carcinomatosis or psychiatric disorders were excluded, as well as patients with history of alcohol abuse or on vitamin supplementation. Moreover, patients treated with antiepileptic drugs, amifostine, glutathione, glutamine, calcium and magnesium solutions and other neuromodulatory agents were not included in the study. Patients affected by diabetes mellitus were enrolled only if neither clinical nor neurophysiologic signs of peripheral nerve involvement were detected. Written informed consent for both electrophysiologic studies and genetic analysis was obtained.

This study has been conducted between April 2006 and May 2007, in accordance with the rules of the local Ethics Committee and the Declaration of Helsinki.

\section{Endpoint of the study}

The study was based on the assumption that acute OXA-neurotoxicity is related to a less common genotype of SK3 gene. Therefore, the primary endpoint of the study was to demonstrate a difference in the incidence of acute neurotoxicity between patients with common and patients with less frequent genotype. 
A detailed neurological evaluation was performed before and after the first OXA administration. It consisted of cranial nerve examination, peripheral sensory and muscle strength testing and deep tendon reflex assessment. The neurophysiologic evaluation included sensory and motor nerve conduction studies combined with the measurement of F-wave latencies. Patients were examined in a warm comfortable room; the skin temperature was maintained above $32^{\circ} \mathrm{C}$. Motor nerve conduction studies were carried out on ulnar, peroneal and tibial nerves. The stimulus was an electrical square pulse of $0.2 \mathrm{~ms}$; current intensity was $150 \%$ of that needed to obtain maximal CMAP amplitude (20-40 $\mathrm{mA})$. The position of the recording electrodes was considered optimal when the CMAP showed maximal amplitude and negative onset. As for ulnar nerves, the recording monopolar surface electrode was placed on abductor digiti quinti and referred to the first phalanx of the fifth finger. The CMAP of the tibial nerves was recorded from abductor hallucis and referred to the metatarsal-phalangeal joint of the big toe, while for peroneal nerves it was recorded from extensor digitorum brevis and referred to the metatarsal-phalangeal joint of the little toe. Sensory nerve conduction studies were carried out on the sural nerve. It was stimulated antidromically: the recording surface electrode was placed under the lateral malleolus bone and the stimulating electrode was kept at a distance of $12 \mathrm{cms}$ from the active recording electrode. Needle electromyography (EMG) was performed on gastrocnemius by using concentric needle electrodes.

In selected cases these studies were repeated after subsequent OXA administrations in the course of treatment.

\section{Genetic analysis (of SK3 polymorphism)}

A venous blood sample was obtained from each patient before the first OXA treatment. Peripheral blood mononuclear cells were isolated from whole blood under sterile conditions using Lymphoprep (Axis-Shield PoC , Norton, MA, USA) and genomic DNA was isolated by a standard salting out procedure [19]. Polymerase chain reaction (PCR) amplification of the second KCNN3 CAG polymorphism was adapted from the method described by Austin and co-workers [20]. CAGcontaining fragments were amplified from genomic DNA using the primers KCNN3-I-F 5'- 
CAGCAGCCCCTGGGACCCTCGC and KCNN3-I-R 5'-GGAGTTGGGCGAGCTGAGACAG.

PCR was performed with QIAGEN master mix (Qiagen, Hilden, Germany), according to the manufacturer's instructions. PCR parameters consisted of 1 cycle at $95^{\circ} \mathrm{C}$ for $4 \mathrm{~min}, 35$ cycles at $94^{\circ} \mathrm{C}$ for $1 \mathrm{~min}$, primer annealing at $55^{\circ} \mathrm{C}$ for $1 \mathrm{~min}$ and extension at $72^{\circ} \mathrm{C}$ for $30 \mathrm{sec}$. Final PCR extension was performed at $72^{\circ} \mathrm{C}$ for $2 \min$ [20]. PCR products were analysed on polyacrylamide gel after staining with Ethidium Bromide. Each allele was isolated and extracted by polyacrylamide gel with QIAquick Gel Extraction Kit and sequenced on an ABI PRISM 310 Genetic Analyzer.

\section{Statistical analysis}

Considering that about $60 \%$ of Caucasian have 16-19 CAGs allele of SK3 gene, we assumed a $10 \%$ incidence of neurotoxicity in these patients and hypothesized at least a $50 \%$ incidence of neurotoxicity in patients with a less common genotype. The sample size required to achieve a study power of $80 \%$ was at least 15 patients for each group. Among the less common genotype, both longer and shorter alleles were included, therefore three groups were also considered for further statistical analysis: patients with the most common alleles (16-19 CAGs), patients with longer alleles (> 19 CAGs) and patients with shorter alleles (<16 CAGs).

Statistical analysis was performed using SPSS15 software. Differences in the distribution of categorical variables were assessed using Pearson's chi-square test. Relationships between continuous variables were analysed by linear regression analysis. Severe acute neurotoxicity was set as the dependent variable of a multivariate logistic regression analysis. Goodness of fit was assessed using the Hosmer and Lemeshow test. The significance level was set at $p=0.05$ for each test. 


\section{Results}

Patients

Forty patients were enrolled in this study; their characteristics are summarised in Table 1. Most of them were male (62.5\%). Median age was 59 years. ECOG performance status (PS) was zero in most patients (85\%) and 1 in the remaining 15\%. OXA was used in combination with 5-Fluorouracil and Leucovorin; OXA dosage was $100 \mathrm{mg} / \mathrm{m}^{2}$ in patients with advanced disease (FOLFOX 6 regimen), but $85 \mathrm{mg} / \mathrm{m}^{2}$ in those treated in an adjuvant setting (FOLFOX 4 regimen).

\section{Clinical and neurophysiologic findings}

Before starting chemotherapy the neurological examination was normal in all patients. Twenty-eight out of 40 patients (70.0\%) experienced cold-induced paraesthesias involving both hands and feet after the first dose of OXA. Among these patients, 17 (42.5\%) suffered from a few, mild and self-limiting episodes of paraesthesia while $11(27.5 \%)$ complained of intense cold-induced paraesthesias, also involving the perioral area, associated with muscle cramps and visible fasciculations in two cases (5.0\%), jaw tightness in two (5.0\%), lack of a portion of the visual field in one (2.5\%) and ptosis in the left eye in another patient (2.5\%). According to this clinical evaluation, acute neurotoxicity was absent in 12 patients (G0-clinical), mild in 17 (G1-clinical) and severe in 11 (G2-clinical). Neurophysiologic studies performed 24 hours after the first OXA administration showed the presence of repetitive components of the CMAP in all but four patients complaining of symptoms and signs of nerve hyperexcitability. Interestingly, we observed differences in amplitude and distribution of the motor nerve repetitive components, which in most cases matched with the clinical phenotype.

In particular, in 15 patients we recorded the presence of a few repetitive components of the CMAP, usually restricted to a single motor nerve (more often tibial nerves) (Fig.1a). The EMG examination of both gastrocnemius muscles was normal.

Conversely, in 9 other patients the repetitive components of the CMAP were much more evident. They were also more widely distributed, being detected in each of the nerves examined (Fig. 1b). In these patients the EMG showed fasciculation potentials, doublet, triplet or multiple single-unit discharges 
with a high irregular intraburst frequency. In addition, after nerve stimulation, as following voluntary muscle contraction, an asynchronous contraction of single or multiple motor units was observed, producing myokymia. The occurrence of afterdischarges and, in a single case, of cramps in the gastrocnemius, was also recorded. No sensory nerve abnormalities were found.

The neurophysiologic examination was normal in all 16 remaining cases. The patients were divided into three groups according to these neurophysiologic findings: G0-neurophysiological (16 patients); G1-neurophysiological (15 patients) and G2-neurophysiological (9 patients).

The three most severely impaired patients in the G2-neurophysiological (G2-NP) group were also examined before and after the third, fifth and last treatment courses. Before each cycle, the repetitive CMAP components continued to be recorded, although they were reduced in number and amplitude. After each OXA administration a progressive and pronounced increase in the number, amplitude and distribution of CMAP repetitive components was observed; at the same time needle EMG documented spontaneous muscle activity (Fig. 2).

A single G0-clinical patient, initially included in the G0-NP group, complained of muscle cramps and distal paraesthesias only after the twelfth course of chemotherapy and was again submitted to neurophysiological examination. The new evaluation after the last OXA administration revealed striking signs of nerve hyperexcitability, which were completely absent at the first assessment (Fig. 3).

\section{Correlation between clinical examination and neurophysiologic findings}

In all patients experiencing no neurological symptoms (G0-clinical), NCS and EMG were normal (G0-NP). Most patients (9 out of 11) experiencing severe acute toxicity (G2-clinical) had neurophysiologic signs of severe damage (G2-NP). The remaining two G2-clinical patients had neurophysiologic signs of mild (1 patient) or no (1 case) nerve hyperexcitability. Only three G1-clinical patients were G0 at nerve conduction studies, whereas the other fourteen G1-clinical patients showed neurophysiologic findings of G1-NP as well. 
Thus a close correlation between clinical and neurophysiologic findings (78 to100\%) was observed. Therefore, in order to ensure accuracy and reproducibility, the neurophysiologic classification was used for the statistical comparison with the genotype analysis.

\section{Genotype}

Analysis of the highly polymorphic CAG motif of SK3 resulted in 9 different alleles with a number of repeat units (repetitive sequences) ranging between 12 and 23 CAGs (Fig 4). In accordance with the prevalence described among Caucasians $[16,17]$, the most commonly found alleles in our patients were allele 19 and allele 18, detected in 16 and 15 patients, respectively. Fewer than a third of patients were homozygous (13 patients), while the remaining 27 were heterozygous.

Table 2 shows the distribution of allele frequencies and their correlation with either acute neuro-toxicty (both G1 and G2-NP) or absence of acute toxicity (G0-NP). It was not possible to analyze the distribution of neurotoxicity in relation to the different haplotypes, since the number of haplotypes was too large in comparison to sample size; thus association analysis was made considering the incidence of neurotoxicity in relation to each single allele of the haplotype.

When G2-NP patients were compared with G0-NP and G1-NP patients, a significant difference in genotype frequencies was observed, alleles 13 and 14 being much more frequent in G2-NP patients (chi-square 48.6; df:16; $\mathrm{p}=.0001$ ). Five out of the nine G2-NP patients were homozygous for alleles 13 or 14 , two G2-NP patients were heterozygous, showing allele 14 together with either allele 17 or allele 19, and another G2-NP patient was heterozygous for allele 15.

When the patients were divided into three groups according to alleles 13 to 15,16 to 19 and 20 to 23 , respectively, a significantly higher incidence of G2-NP acute toxicity was observed in the first group than in either of the other groups (chi-square 36.9; df:4; $\mathrm{p}=.0001$ ). No statistically significant differences in allele distribution were found when G0-NP and G1-NP patients were compared. The incidence of G2-NP toxicity was not significantly different in patients treated at $85 \mathrm{mg} / \mathrm{m}^{2}$ in comparison to those treated at $100 \mathrm{mg} / \mathrm{m}^{2}$; to this regard, it should be noticed that two G2-NP patients were treated with the lower dose. 
The linear regression analysis of allele distribution showed no threshold effect, meaning that there is no critical number of CAG repeats below which the incidence of OXA-related acute neurotoxicity exponentially increases. Conversely, there is an inverse linear ratio between the number of CAG repeats and the occurrence of toxicity $(B=-0.152 ; \mathrm{p}<0.001)$, with lower numbers of CAG motifs correlating with more frequent acute neurotoxicity.

Severe acute neurotoxicity (G2-NP patients) was set as the dependent variable in a backward stepwise multivariate logistic regression model, in which the length of alleles and other clinical variables, including sex (M vs F), age ( $\leq 55$ vs $>55$ ), diabetes (yes vs no) and previous chemotherapy (yes vs no), were entered as independent variables. G2-NP toxicity was predicted by the presence of diabetes $(\mathrm{OR}=$ 9.764, 95\%CI: $1.187-80.317$; p 0.034), while patients carrying alleles with a high number of CAGs were protected from neurotoxicity $(\mathrm{OR}=0.381,95 \% \mathrm{CI}: 0.247-0.590 ; \mathrm{p} 0.001)($ Table $\mathrm{n} 3)$. The model adequately fit the data according to the Hosmer and Lemeshow test (Chi-square 4.806, p 0.684; $90 \%$ of patients correctly classified).

\section{Allergic-type reactions to Oxaliplatin}

During the course of the study four patients developed allergic-type reactions to oxaliplatin, leading to interruption of the treatment. Two patients experienced intense dyspnoea and bronchospasm, one developed an intense cutaneous erythema and another severe hypotension with a transient lack of consciousness. Interestingly, all these patients belonged to the G2-NP group. Their genotype was characterized by a short number of repeats on the polymorphic trait of the SK3 gene (13-15 CAGs).

\section{Oxaliplatin clinical efficacy}

Thirteen patients were treated in an adjuvant setting and thus they were not evaluable for response. Among the remaining 27 patients, six were affected by pancreatic or bile ducts cancers and all of them experienced disease progression during chemotherapy. Out of 21 colorectal cancer patients, nine 
experienced a partial response, six a disease stabilization and the remaining six a progression. No difference in SK3 polymorphisms was observed between responders and not-responders. 


\section{Discussion}

Peripheral neurotoxicity impairs patients' daily activities and may lead to the withdrawal of OXA even in responding patients, thus negatively affecting the efficacy of chemotherapy. The occurrence of this complication is a challenge for oncologists, because its treatment gives largely disappointing results. Pre-treatment with $\mathrm{Ca}^{++} / \mathrm{Mg}^{++}$may reduce the frequency of neurotoxicity, but may also decrease the clinical efficacy of the drug [21].

Different mechanisms appear to be responsible for both chronic and acute forms of neurotoxicity induced by platinum derivatives. The first is probably related to an axonopathy due to the accumulation of platinum compounds within the dorsal root ganglia, the second to a transient impairment of ion channel function, but there is still no agreement about which channel is involved [68, 11-13], neither about the possible correlation between acute and chronic neurotoxocity.

In the mouse hemidiaphragm, the effect of OXA can be prevented by VGNaC blockers, such as TTX, and it is not replicated by apamin, a VGKC blocker, suggesting that OXA acts by altering the activity of VGNaCs [12].

A possible interference of OXA with $\mathrm{VGNaCs}$ has also been suggested by the fact that in the rat sural nerve OXA induces a decrease in the amplitude and duration of the action potential as well as a lengthening of the refractory period of peripheral nerves [11]. The recovery cycle in human motor axons is composed of absolute and relative refractory periods, followed by supernormal (decreased threshold) and subnormal (increased threshold) periods. While the inactivation rate of $\mathrm{Na}^{+}$channels is the main factor influencing the supernormal period, the subnormal period is regulated by $\mathrm{K}^{+}$channels [22]. These experimental observations, combined with the known similarities between neuromyotonia and acute OXA-induced neurotoxicity and with recent observation that SK3 subtypes of potassium channels may be apamin-insensitive [23], led us to hypothesise a different mechanism for acute OXA neurotoxicity, involving in particular the SK3 subtype of KCa.

Therefore in patients treated with OXA we investigated the clinical-genetic correlation between the polymorphic CAG repeat motif within exon 1 of the SK3 gene, which corresponds to the second Cterminal polyglutamine array in the SK3-protein, and the occurrence of acute neurological toxicity, as 
assessed by electrophysiologic means. A correlation was found between clinically and electrophysiologically assessed neurotoxicity, and the most accurate and reproducible NP-groups were therefore used for comparison with the genetic analysis. The study results show that patients carrying a short CAG repeats allele (13-15 repeats) experienced a significantly higher incidence of severe acute OXA-induced neurotoxicity when compared with patients carrying longer alleles. Interestingly, two G2-NP patients were treated with the lower dose of $85 \mathrm{mg} / \mathrm{m}^{2}$, suggesting that SK3 polymorphism more than OXA dosage would be related to neurotoxocity.

Despite the small number of patients in our sample, the results are statistically significant allowing us to suggest that OXA might display its toxic effect by interfering with SK3 function, possibly impairing the formation of tetramers when a short polyglutamine tail is present. As a consequence, in the peripheral nerve terminals, SK3 channels would not contribute to after-hyperpolarisation following the action potential, thus causing nerve hyperexcitability. This condition appears as afterdischarges, fasciculation potentials, and doublet, triplet or multiple single-unit discharges combined with stable repetitive components of CMAP, as occurred in patients affected by neuromyotonia [9, 10, 24]. Moreover, SK3 channels seem to have a certain role in the release of histamine by mast cells [25]. This observation might be relevant in relation to the so called "allergic-type" reactions to OXA. In our study four patients had this type of allergic reaction to OXA, leading to drug discontinuation. Interestingly enough, all of them carried a short SK3 allele (13-15 CAGs) and belonged to the G2-NP group. Based on the recent observation that the mechanism of this potentially life-threatening reaction could not be immunoglobulin-mediated [26-27], we may speculate that also these reactions would be related to a dysfunction of SK3, giving us the opportunity to identify patients at risk before exposure to OXA. In the same way, based on our statistically significant results in the cohort of patients examined, we would identify in advance patients with a bias towards the occurrence of acute OXA neurotoxicity. This would be extremely useful considering the wide use of OXA in adjuvant combination therapy for colon cancer.

No relationship emerged between chemotherapy efficacy and SK3 polymorphic polyglutamine trait. However it should be emphasized that the study was not aimed to this endpoint, that probably could be 
better addressed by assessing the expression/polymorphisms of SK3 protein in the tumor tissue more than in the peripheral blood mononuclear cells.

In conclusion, even if there is a need of functional studies in order to confirm these preliminary observations, this pilot study shows the presence of a genetic predisposition to develop acute OXA neurotoxicity and suggests a new fascinating mechanism of interaction between OXA and the smallconductance calcium-dependent potassium channel of the peripheral nervous system. 


\section{References}

1. Culy CR, Clemett D, Wiseman LR (2000). Oxaliplatin: a review of its pharmacological properties and clinical efficacy in metastatic colorectal cancer and its potential in other malignancies. Drugs 60: 895-924

2. de Gramont A, Figer A, Seymour H, Homerin M, HmissiA, Cassidy J, Boni C, Cortes-Funes H, Cervantes A, Freyer G, Papamichael D, Le Bail N, Louvet C, Hendler D, de Braud F, Wilson C, Morvan F, Bonetti A (2000). Leucovorin and fluorouracil with or without oxaliplatin as first-line treatment in advanced colorectal cancer. J Clin Oncol 18: 2938-2947

3. Goldberg RM, Sargent DJ, Morton RF, Fuchs CS, Ramanathan RK, Williamson SK, Findlay BP, Pilot HC, Alberts SR (2004). A randomized controlled trial of fluorouracil plus leucovorin, irinotecan and oxaliplatin combinations in patients with previously untreated metastatic colorectal cancer. J Clin Oncol 22: 23-30

4. Gamelin E, Gamelin L, Bossi L and Quasthoff S (2002). Clinical aspects and molecular basis of oxaliplatin neurotoxicity: current management and development of preventive measures. Semin Oncol 29: 21-33.

5. Cassidy J and Misset JL (2002). Oxaliplatin-related side-effects: characteristics and management. Semin Oncol 29: 11-20

6. Screnci D, McKeage M (1999). Platinum neurotoxicity: clinical profiles, experimental models and neuroprotective approaches. J Inorg Biochem 77: 105-110.

7. Wilson RH, Lehky T, Rebecca RT, Quinn MG, Floeter MK, Grem JL (2002). Acute oxaliplatininduced peripheral nerve hyperexcitability. J Clin Oncol 20: 1767-1774. 
8. Lehky TJ, Leonard GD, Wilson RH, Grem JL and Floeter MK (2004). Oxaliplatin-induced neurotoxicity: acute hyperexcitability and chronic neuropathy. Muscle Nerve 29: 387-392.

9. Hart IK Maddison P, Newsom-Davis J, Vincent A, Mills KR (2002) Phenotypic variants of autoimmune perypheral nerve hyperexcitability. Brain 125: 1887-95

10. Hart IK, Waters C, Vincent A, Newland C, Beeson D, Pongs O, Morris C, Newsom-Davis J (1997). Autoantibodies detected to expressed K+ channels are implicated in neuromyotonia. Ann Neurol 41: 238-246.

11. Adelsberger H, Quasthoff S, Grosskreutz J, Lepier A, Eckel F, Lersch C (2000). The chemotherapeutic oxaliplatin alters voltage-gated $\mathrm{Na}(+)$ channel kinetics on rat sensory neurons. Eur $\mathrm{J}$ Pharmacol 406: 25-32.

12. Webster RG, Brain KL, Wilson RH, Grem JL and Vincent A (2005). Oxaliplatin induces hyperexcitability at motor and autonomic neuromuscular junctions through effects on voltage-gated sodium channels. British Journal of Pharmacology 146: 1027-1039.

13. Grolleau F, Gamelin L, Boisdron-Celle M, Lapied B, Phelate M, Gamelin E (2001). A possible explanation for a neurotoxic effect of the anticancer agent oxaliplatin on neuronal voltage-gated sodium channels. J Neurophysiol 85: 2293-2297.

14. Basso M, Cassano A, Modoni A, Spada D, Trigila N, Quirino M, Schinzari G, Barone C (2008). A reversibile coma after oxaliplatin administration suggests a pathogenetic role of electrolyte imbalance. Eur J Clin Pharmacol 64: 739-741. 
15. Kimura T, Takahashi MP, Fujimura H, Sakoda S (2003). Expression and distribution of a smallconductance calcium-activated potassium channel (SK3) protein in skeletal muscles from myotonic muscular dystrophy patients and congenital myotonic mice. Neurosci Lett 347: 191-195

16. Wittekindt O, Jauch A, Burgert E, Scharer L, Holtgreve-Grez H, Yvert G, Imbert G, Zimmer J, Hoehe MR, Macher JP, Chiaroni P, van Calker D, Crocq MA, Morris-Rosendahl DJ (1998). The human small conductance calcium-regulated potassium channel gene (hSKCa3) contains two CAG repeats in exon 1, is on chromosome 1q21.3 and shows a possible association with schizophrenia. Neurogenetics 1: 259-265.

17. Chandy KG, Fantino E, Wittekindt O, Kalman K, Tong LL, Ho TH, Gutman GA Crocq MA, Ganguli R, Nimgaonkar V, Morris-Rosedahl DJ, Gargus JJ (1998). Isolation of a novel potassium channel gene hSKCa3 containing a polymorphic CAG repeat: a candicate gene for schizophrenia and bipola disorder. Mol Psychiatry 3: 32-37.

18. Frei E, Spindler I, Grissmer S and Jager H (2006). Interactions of N-terminal and C-terminal parts of the small conductance $\mathrm{Ca}^{2+}$ activated $\mathrm{K}^{+}$channel, $h$ SK3. Cell Physiol Biochem 18: $165-176$.

19. Miller SA, Dykes DD, Plensky HF (1988). A simple salting out procedure for extracting DNA from human nucleated cells. Nucleic Acid Res 16: 1215.

20. Austin CP, Holder DJ, Ma L, Mixson LA, Caskey CT (1999). Mapping of hKCa3 to chromosome 1q21 and investigation of linkage of CAG repeat polymorphism to schizophrenia. Mol Psychiatry 4: $261-266$ 
21. Grothey A, Hart LL, Rowland KM, Ansari RH, Alberts SR, Chowhan NM, Shpilsky A, Hochster HS (2008). Intermittent oxaliplatin (oxali) administration and time-to-treatment failure (TTF) in metastatic colorectal cancer (mCRC): Final results of the phase III CONcePT trial. J Clin Oncol 26: abstract 4010 .

22. McIntyre CC, Richardson AG and Grill WM (2002). Modeling the excitability of mammalian nerve fibers: influence of afterpotentials on recovery cycle. J Neurophysiol 87: 995-1006

23. Wittekindt OH, Visan V, Tomita H, Imtiaz F, Gargus JJ, Lehmann-Horn F, Grissmer S, MorrisRosendahl DJ (2004). An apamin- and scyllatoxin-insensitive isoform of the human SK3 channel. Mol Pharmacol 65: 788-801.

24. Tomimitsu H, Kimiyoshi A, Nagado T, Watanabe O, Otsuka R, Kurono A, Sonoda Y, Osame M, KameyamaM (2004). Mechanism of action of voltage-gated $\mathrm{K}^{+}$channels antibodies in acquired neuromyotonia. Ann Neurol 56: 440-444.

25. Mark Duffy S, Berger P, Cruse G, Yang W, Bolton SJ, Bradding P (2004). The $\mathrm{K}^{+}$channels iKCA1 potentiates $\mathrm{Ca} 2+$ influx and degranulation in human lung mast cells. J Allergy Clin Immunol 114: 66-72

26. Maindrault-Goebel F, Andrè T, Tournigand C, Louvet C, Perez-Staub N, Zaghib N, de Gramont A (2005). Allergic-type reactions to oxaliplatin: retrospective analysis of 42 patinets. Eur J Cancer 41: 2262-2267. Epub 2005 Sep 9

27. Siu SW, Chan RT, Au GK (2006). Hypersensitivity reactions to oxaliplatin: experience in a single institute. Ann Oncol 17: 259-261. Epub 2005 Nov 9. 
Table n 1. Patients' characteristics (n 40)

Median age

Ethnicity

Caucasian

Male/Female

Performance status (ECOG)

Zero

35

One

Tumor*

Colorectal cancer

Pancreatic cancer

Bile ducts cancer

* no gastric cancer patients were enrolled due to comorbidities potentially responsible for peripheral nerve impairment
$40(100 \%)$

$25 / 15$
34 (13 adjuvant)

5

1

Line of therapy

First

Second

11 
Table $\mathbf{n}$ 2. Incidence of acute OXA-related neurotoxicity and allelic polymorphisms of SK3 channel.

OXA-related acute neuro-toxicity
Grade $0(n)$
Grade 1 (n)
Grade 2 (n)

Alleles

13

0

0

6

14

1

0

6

15

1

2

1

16

3

2

0

17

3

3

2

18

10

7

1

19

10

9

1

20

3

5

1

23

1

2

0 
Table n 3: Multivariate logistic regression: the final model.

\section{OR}

0.381

Alleles

$(\leq 15 />15)$

0.001

$0.247-0.590$

\section{Diabetes}

$(\mathrm{Y} / \mathrm{N})$

Age

3.122

$0.505-19.287$

0.222

$(\leq 55 />55)$ 


\section{Legend to figures}

Figure 1a: Motor NCS in a G1-NP patient: tibial nerve.

A series of CMAP repetitive components recorded from abductor hallucis following tibial nerve stimulation after OXA infusion. The screen is split with lower sensitivity ( $5 \mathrm{mV} / \mathrm{Division})$ to the left of the dark vertical line, to show the full initial CMAP generated by distal stimulation, and higher sensitivity (0.2 $\mathrm{mV} / \mathrm{Division})$ to the right, to allow visualization of some repetitive components of the CMAP. The sweep speed is $10 \mathrm{msec} /$ Division.

Figure 1b: Motor NCS in a G2-NP patient: ulnar nerve A series of CMAP repetitive components recorded from abductor hallucis following tibial nerve stimulation after OXA infusion. The screen is split with lower sensitivity ( $5 \mathrm{mV} / \mathrm{Division})$ to the left of the dark vertical line, to show the full initial CMAP generated by distal stimulation, and higher sensitivity ( $0.5 \mathrm{mV} / \mathrm{Division})$ to the right, to allow visualization of the numerous repetitive components of the CMAP (black arrow). The sweep speed is $10 \mathrm{msec} /$ Division.

Figure 2: The figure shows motor NCS (tibial nerve) in one G2-NPpatient, before (a) and after (b) the third cycle and before (c) and after (d) the fifth cycle, respectively. Before each administration the CMAP repetitive components continued to be recorded, though reduced in number and amplitude. After OXA infusion a significant increase in their number, amplitude and distribution is evident.

Figure 3: In a single patient NCS were normal after the first cycle (a:tibial nerve), while striking signs of nerve hyperexcitability were recorded after the XII course of OXA (b, c: tibial and peroneal nerve, respectively). At EMG study performed on gastrocnemius a cramp was recorded (d).

Figure 4: SK3 polymorphic CAG motif within exon 1. (a) upper panel: allele containing a long repeat trait (19 CAG). (b) lower panel: allele containing a short repeat trait (13 CAG) 

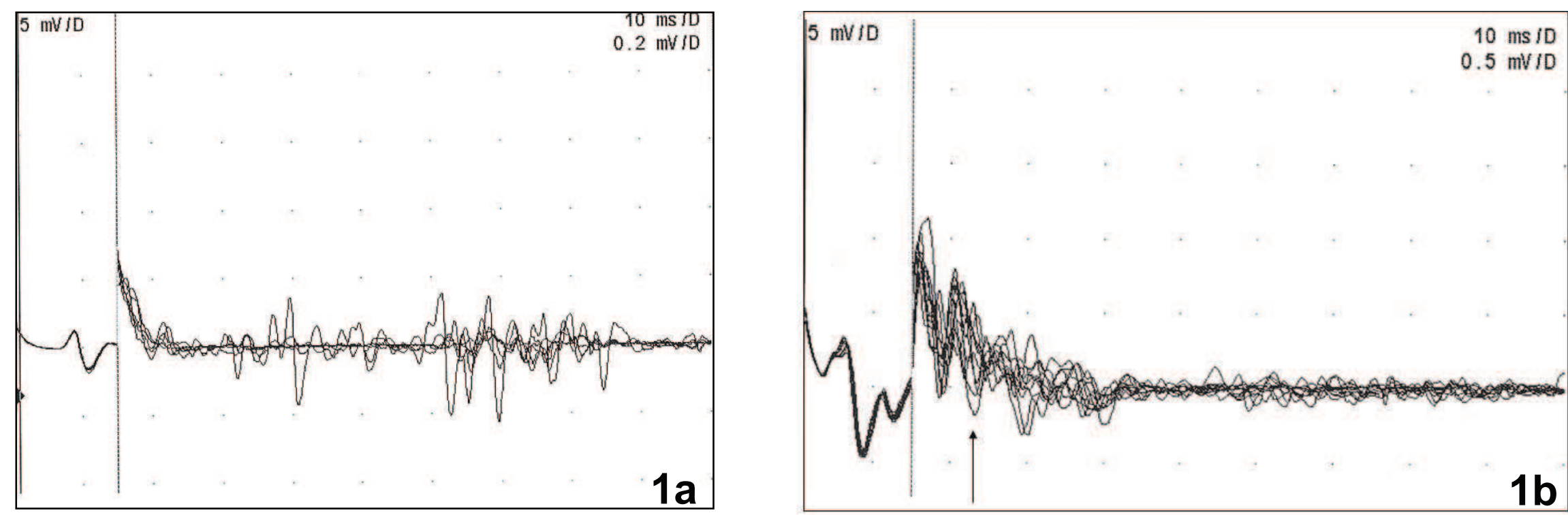

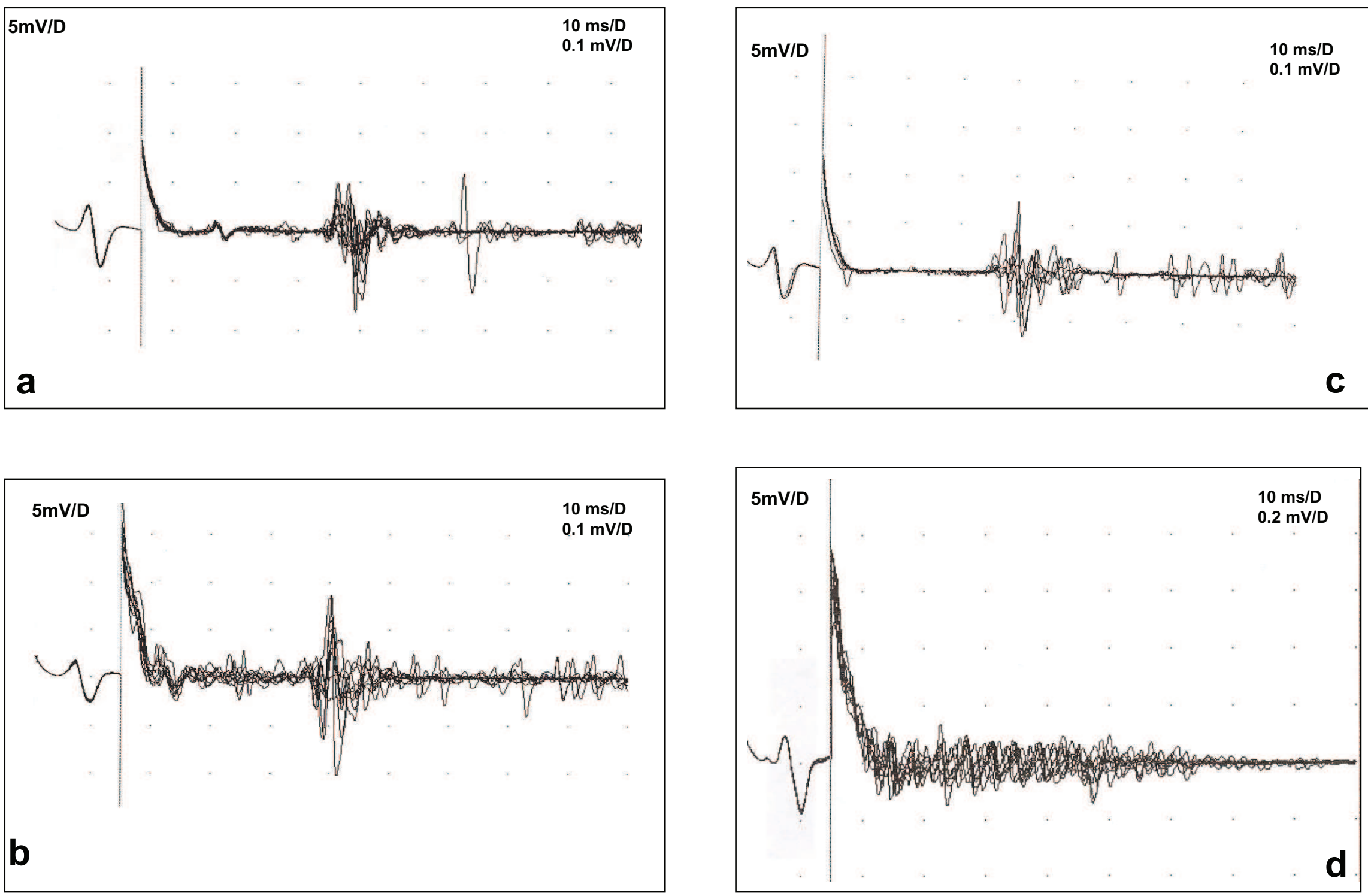

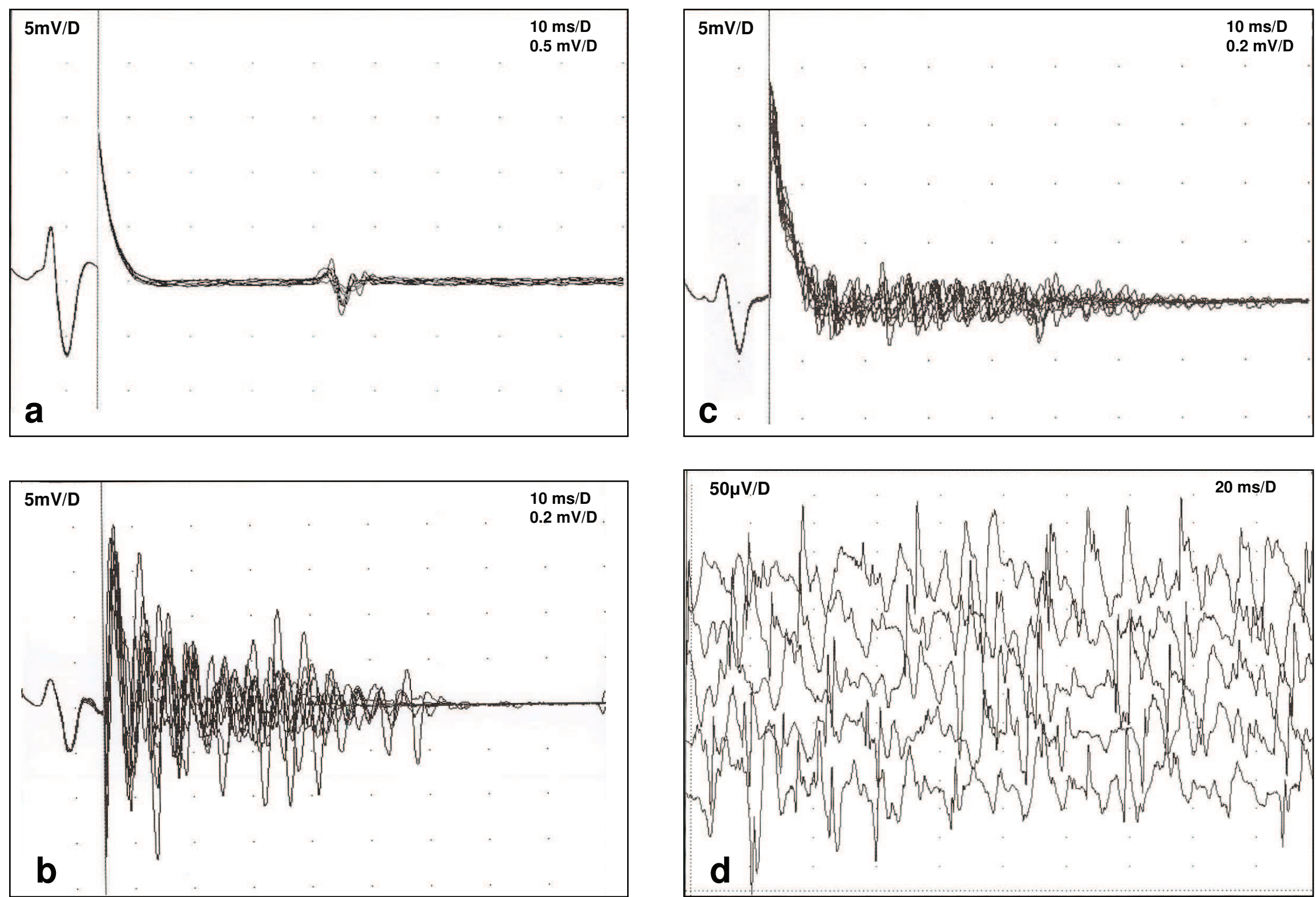


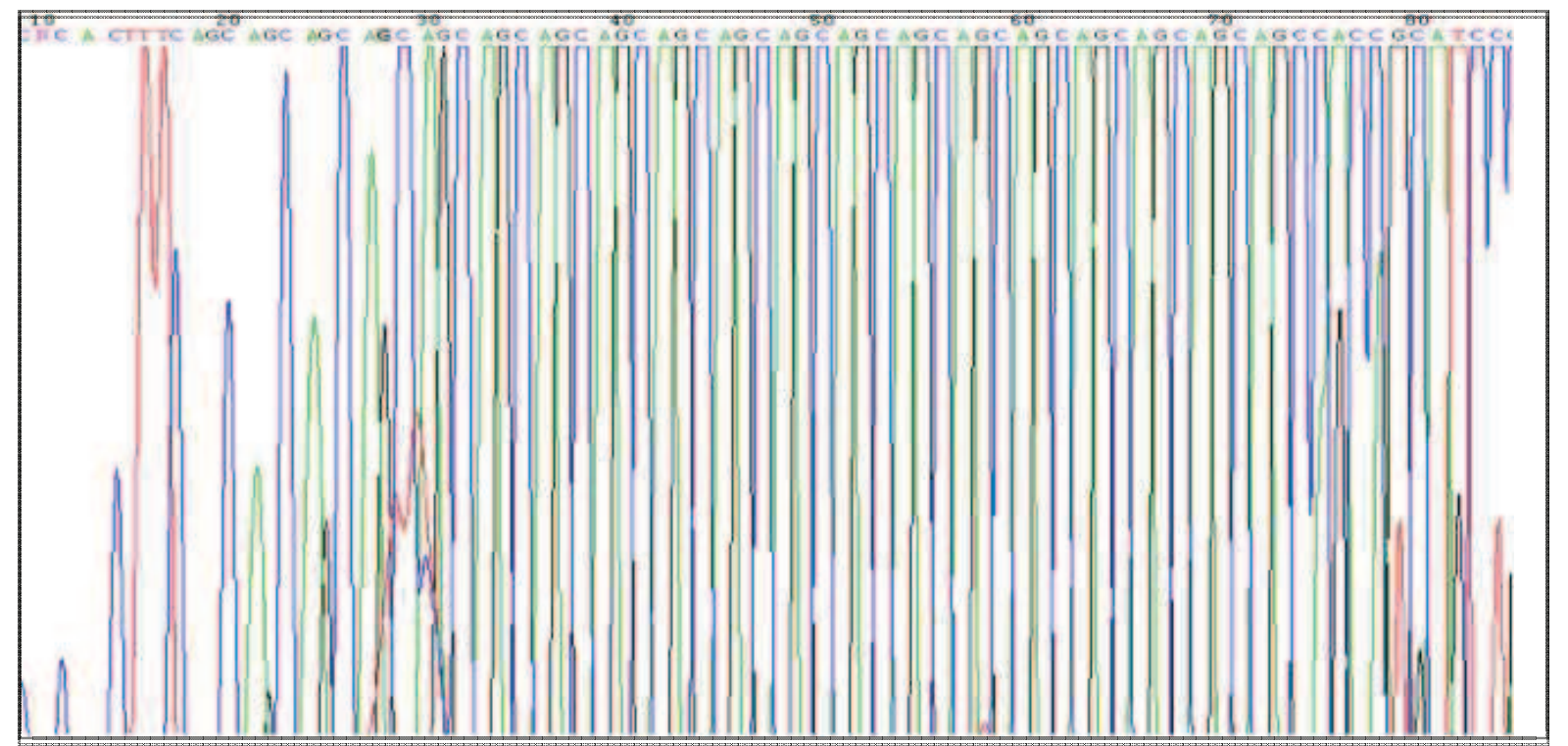

a

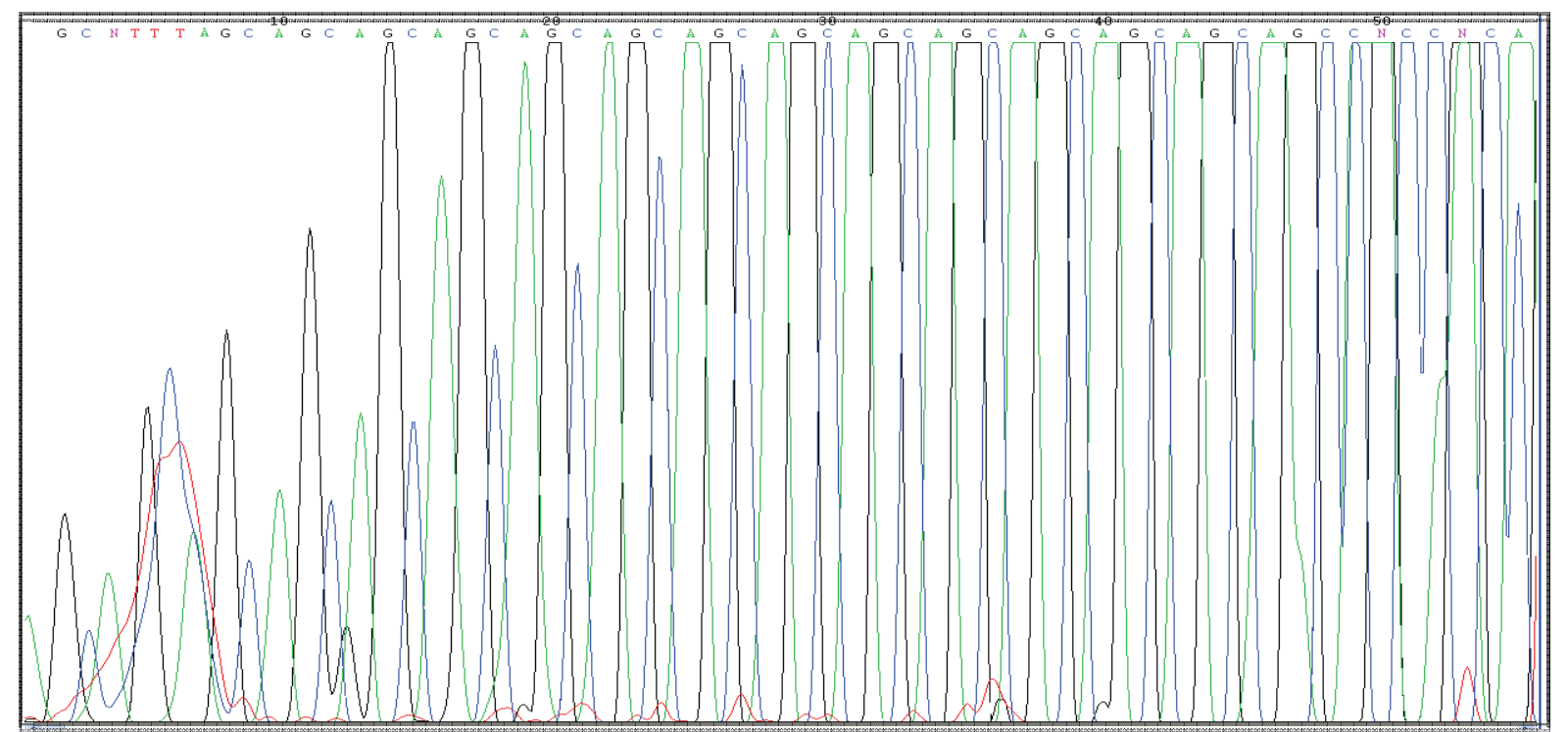

b 